\title{
Linearly Coupled Bose-Einstein Condensates: From Rabi Oscillations and Quasi-Periodic Solutions to Oscillating Domain Walls and Spiral Waves
}

\author{
B. Deconinck ${ }^{1}$, P.G. Kevrekidis ${ }^{2}$, H.E. Nistazakis ${ }^{3}$ and D.J. Frantzeskakis ${ }^{3}$ \\ ${ }^{1}$ Department of Applied Mathematics, University of Washington, Seattle, WA 98195, USA \\ ${ }^{2}$ Department of Mathematics and Statistics, University of Massachusetts, Amherst MA 01003-4515, USA \\ ${ }^{3}$ Department of Physics, University of Athens, Panepistimiopolis, Zografos, Athens 15784, Greece
}

In this paper, an exact unitary transformation is examined that allows for the construction of solutions of coupled nonlinear Schrödinger equations with additional linear field coupling, from solutions of the problem where this linear coupling is absent. The most general case where the transformation is applicable is identified. We then focus on the most important special case, namely the well-known Manakov system, which is known to be relevant for applications in Bose-Einstein condensates consisting of different hyperfine states of ${ }^{87} \mathrm{Rb}$. In essence, the transformation constitutes a distributed, nonlinear as well as multi-component generalization of the Rabi oscillations between two-level atomic systems. It is used here to derive a host of periodic and quasi-periodic solutions including temporally oscillating domain walls and spiral waves.

Introduction. The recent progress in experimental and theoretical studies of Bose-Einstein condensates (BECs) [1] has made solitary matter waves physically relevant objects. One-dimensional (1D) dark [2] and bright [3] solitons have been observed in recent experiments. On the other hand, optical solitons have a time-honored history as fundamental nonlinear excitations in optical fibers and waveguides (see, e.g., the recent reviews $[4,5]$ ).

A very relevant generalization of this class of physical systems and of the solitary waves they can support concerns the case of multiple coupled components. There has recently been a considerable volume of work relevant to the properties of coupled BECs ranging from the ground state solutions $[6,7]$ to the small-amplitude excitations [8] of the order parameters. Furthermore, the formation of various structures including domain walls $[9,10]$, bound dark-dark [11], dark-bright [12], dark-antidark, dark-gray, bright-antidark and bright-gray soliton complexes [13], as well as spatially periodic states [14] was also predicted. On the other hand, experimental results have been reported for mixtures of different spin states of ${ }^{87} \mathrm{Rb}[15]$ and mixed condensates $[16,17]$. It is relevant to mention the efforts into the realization of two-component BECs from different atomic species, such as ${ }^{41} \mathrm{~K}-{ }^{87} \mathrm{Rb}$ [18] and ${ }^{7} \mathrm{Li}-{ }^{133} \mathrm{Cs}$ [19].

Typically, the relevant model for two coupled BECs involves two nonlinearly coupled Gross-Pitaevskii (GP) equations. However, in experiments with a radiofrequency (or an electric field) coupling two separate hyperfine states $[15,20]$, the relevant model involves also a linear coupling between the wavefunctions. The governing normalized equations are then of the form:

$\begin{aligned} i \psi_{1 t} & =\left[-\frac{1}{2} \Delta+V+a_{11}\left|\psi_{1}\right|^{2}+a_{12}\left|\psi_{2}\right|^{2}\right] \psi_{1}+\alpha \psi_{2}, \\ i \psi_{2 t} & =\left[-\frac{1}{2} \Delta+V+a_{12}\left|\psi_{1}\right|^{2}+a_{22}\left|\psi_{2}\right|^{2}\right] \psi_{2}+\alpha \psi_{1},\end{aligned}$

where $V \equiv V(r)$ is the relevant potential, typically consisting of a magnetic trap and/or an optical lattice [1,21], while $\psi_{j}$ 's represent the condensate wavefunctions. The intra- and inter-species interactions are characterized by the coefficients $a_{j j}(j=1,2)$ and $a_{12}$ respectively, while $\alpha$ denotes the strength of the radio-frequency (or electric field) coupling. Note that Eqs. (1)-(2), combining both linear and nonlinear couplings, occur in fiber optics as well: In that case, $\psi_{j}$ 's are two coupled electric field envelopes of the same wavelength but of different polarizations and the linear coupling is generated either by a twist applied to the fiber in the case of two linear polarizations, or by an elliptic deformation of the fiber's core in the case of circular polarizations $[22,23]$ (linear coupling is impossible when considering waves of different wavelengths). Another optical model, with only linear coupling between two modes, applies to nonlinear fiber couplers [24] or dual-core nonlinear fibers (see, e.g., [25]). In the context of BECs, this coupling has been recently examined for extended wave solutions in [26].

In the present work, we aim to study a unitary transformation in the context of Eqs. (1)-(2) that completely absorbs the linear coupling between the components into an oscillatory temporal dependence. We illustrate the value of this transformation in a two-fold way: on the one hand, we use it to understand the role of the linear coupling between the components as a means of creating Rabi oscillations between the matter present in the two components (e.g., hyperfine states) [27] and their analog in power oscillations between polarizations in optical systems. On the other hand, we use it to construct exact time-periodic solutions of such linearly coupled nonlinear Schrödinger equations. This way, we identify time-periodic Thomas-Fermi clouds and extended waves in one spatial dimension, as well as sloshing domain walls and vortices in two dimensions. Since the transformation is exact only in the so-called Manakov case of $a_{11}=a_{22}=a_{12}$ [28], we investigate numerically what happens to the constructed time-periodic solutions in cases (relevant to ${ }^{87} \mathrm{Rb}$ experiments) where $a_{11} \neq a_{22} \neq a_{12}$. Finally, we generalize the transfor- 
mation to show that analogous constructions are feasible with a higher number of components. Our focus here is in illustrating the generality and applicability of the transformation to a very broad host of settings (multiple component, as well as higher dimensional cases, also in the presence of external potentials). We should note that special cases of this transformation have previously been identified in optics (in the integrable case of the twocomponent, one-dimensional Manakov solitons in the absence of a potential [23]), as well as in a different format in BECs [in two-component, 1D condensates in the presence of a periodic potential [29], where solutions were constructed as unitary transformations of stationary solutions of the equations (6) below. There the nonstationarity was introduced by the mixing of two stationary solutions that were not in phase.]

Analytical Results. Assuming that $a_{11}=a_{22} \equiv g$ and $a_{12} \equiv h$, Eqs. $(1,2)$ take the following matrix form:

$$
i \psi_{t}-\alpha P \psi=-\frac{1}{2} \Delta \psi+\left(\psi^{\dagger} G \psi\right) \psi+V(\mathbf{x}) \psi,
$$

where

$$
G=\left(\begin{array}{ll}
g & 0 \\
0 & h
\end{array}\right), \quad P=\left(\begin{array}{ll}
0 & 1 \\
1 & 0
\end{array}\right)
$$

Furthermore, in the special case $h=g$ (hence $G=g I$, with $I$ being the identity matrix), we may consider Eq. (3) as a homogeneous equation (LHS) with an inhomogeneous part (RHS); in this case, the solution of the homogeneous equation is given by

$$
\psi=U(t) \psi_{0}=e^{-i \alpha P t} \psi_{0}=\left(\begin{array}{cc}
\cos (\alpha t) & -i \sin (\alpha t) \\
-i \sin (\alpha t) & \cos (\alpha t)
\end{array}\right) \psi_{0} .
$$

Using a variations of parameters approach, we substitute this in the equation for $\psi$. This results in:

$$
i \psi_{0 t}=-\frac{1}{2} \Delta \psi_{0}+\left(\psi_{0}^{\dagger} G \psi_{0}\right) \psi_{0}+V(\mathbf{x}) \psi_{0},
$$

i.e., the same equation as (3), but without the electric field coupling terms. As mentioned above, large classes of exact stationary and nonstationary solutions to this equation were constructed in [9-14,29]. Those solutions can now be used to construct exact solutions to (3). Importantly, stable such solutions, are also relevant to the more general model Eqs. (1)-(2), and in particular to the BEC experiments with ${ }^{87} \mathrm{Rb}$ [16] or ${ }^{23} \mathrm{Na}$ [17]: in that case, the deviations of the values of the nonlinear coefficients $a_{j k}$ from 1 are typically on the order of $3 \%$. Such a small difference acts as a small perturbation and does not alter the stability of the solutions (see also the numerical results below). Note that, typically, these solutions will be nonstationary and, in particular, time-periodic.

The success of this simple variation of parameters method can be phrased differently in physical terms: the equation (6) is invariant under unitary transformations, as it is an additive combination of the linear Schrödinger equation and a nonlinear term with a matrix $G$ that commutes with any matrix. Then the extra linear coupling terms can be removed by using a time-dependent unitary transformation, which does not affect the other terms of the equation. Thus, the solutions of (3) can be thought of as rotating in "spin space".

We should note that more general nonlinear coupling matrices $G$ commute with $U$. In particular:

$$
\tilde{G}=\left(\begin{array}{ll}
0 & 1 \\
1 & 0
\end{array}\right),
$$

also commutes with $U$. The relevant dynamical equations, however, containing the term $\left(\psi_{0}^{\dagger} \tilde{G} \psi_{0}\right) \psi_{0}$ result in nonlinearities of the form $\left|\psi_{1}\right|^{2} \psi_{2}$ and $\psi_{1}^{2} \psi_{2}^{\star}$ in the dynamical equation for $\psi_{1}$ and hence seem less physically relevant.

The density of the different components $n_{i}=\left|\psi_{i}\right|^{2}$ $(i=1,2)$ is given by

$$
\begin{aligned}
n_{1} & =\cos ^{2}(\alpha t)\left|\psi_{01}\right|^{2}+\sin ^{2}(\alpha t)\left|\psi_{02}\right|^{2} \\
& +\left|\psi_{01}\right|\left|\psi_{02}\right| \sin \left(\theta_{2}-\theta_{1}\right) \sin (2 \alpha t), \\
n_{2} & =\cos ^{2}(\alpha t)\left|\psi_{02}\right|^{2}+\sin ^{2}(\alpha t)\left|\psi_{01}\right|^{2} \\
& +\left|\psi_{01}\right|\left|\psi_{02}\right| \sin \left(\theta_{1}-\theta_{2}\right) \sin (2 \alpha t),
\end{aligned}
$$

where $\psi_{01}=\left|\psi_{01}\right| e^{i \theta_{1}}, \psi_{02}=\left|\psi_{02}\right| e^{i \theta_{2}}$. Note that if $\psi_{01}$ and $\psi_{02}$ are time independent, then $n_{1}+n_{2}$ does not depend on time. Thus it is a local constant of the motion (as opposed to the global constant $\int\left(n_{1}+n_{2}\right) d x$ which is conserved for any $\mathrm{G}$ ). This is equivalent to the statement that the nonlinear term in (3) is invariant under the unitary transformation given by $U(t)$. Notice also that the existence of stationary solutions of Eq. (3) requires the expressions (8)-(9) to be independent of $t$, which only happens when both $\psi_{10}$ and $\psi_{20}$ are stationary and equal. Furthermore, clearly the above unitary transformation illustrates that the linear coupling does not affect the integrable nature of the 1D Manakov model (for $V(x)=0$ ).

Numerical Results. We now turn to the practical usefulness of the transformation i.e., constructing timeperiodic solutions of Eq. (3) from stationary solutions of Eq. (6), as well as quasi-periodic solutions of the former from periodic ones of the latter (i.e., the transformation always inserts an additional frequency in the time-dependence of the solution). While one can follow this path also in the absence of the potential for the known, exact solutions of the Manakov model, we will focus herein on the case with the potential, which is more relevant to BECs $[15-17,20]$.

Exact solutions in the presence of a potential are not often available in explicit form for Eq. (6). However, in the presence of the physically relevant, optical lattice potential [30] of the form $V=-V_{0} \sin ^{2}(m x)$ (in one spatial dimension), large classes of such stationary solutions can exist such as [14,31] $\psi_{01}=\left(\sqrt{B_{1}} \cos (m x)-\right.$ $\left.i \sqrt{B_{1}+A_{1}} \sin (m x)\right) e^{-i \omega_{1} t}, \quad \psi_{02}=\left(\sqrt{B_{2}} \cos (m x)-\right.$ 
$\left.i \sqrt{B_{2}+A_{2}} \sin (m x)\right) e^{-i \omega_{2} t}$, where $\omega_{1,2}, A_{1,2}, B_{1,2}$ are appropriate constants. These solutions then become exact, genuinely time-periodic solutions of Eq. (3) according to Eq. (5). Such a solution is given in the top panel of Fig. 1. The bottom panel of the figure illustrates a quasi-periodic solution of Eq. (3), constructed from a periodic one of the form $\psi_{01}=\sqrt{A_{1}} \operatorname{sn}(m x, k) e^{-i \omega_{1} t}$, $\psi_{02}=\sqrt{-A_{2}} \operatorname{cn}(m x, k) e^{-i \omega_{2} t}$ in the elliptic function potential $V=-V_{0} \operatorname{sn}^{2}(m x, k)$, which degenerates into the OL one for $k \rightarrow 0$.

However, typically such explicit solutions are not known, e.g., in the presence of a magnetic trap potential. Then one can use relaxational methods such as a Newton iteration or imaginary time integration to obtain stationary solutions of Eq. (6) and exploit the time-dependence inherent in Eq. (5) to excite exact matter wave oscillations between the two components, producing exact, non-stationary solutions of Eq. (3). A one dimensional example of this strategy is shown in Fig. 2 for the ground state BEC in a magnetic trap potential [1,21] of the form $V(x)=\Omega^{2} x^{2} / 2 . \psi_{2}$ is initialized at an exact stationary solution (in the presence of $V(x)$, obtained via a Newton method), while $\psi_{1}(x, 0)=0$. However, an interesting question then concerns the potential persistence of the Rabi oscillations when the unitary transformation is no longer exact (i.e., for $h \neq g$ ). This is examined in the bottom set of panels in Fig. 2. Clearly, small deviations from the $h=g$ limit (relevant to the BEC context where $a_{11} \approx a_{22} \approx a_{12}$ ) lead to persistence of the matter wave oscillations but with a quasi-periodic beating character. Beyond a critical threshold, however, the oscillations disappear and give rise to chaotic behavior.

Naturally, the same idea for constructing nonstationary solutions can be carried over to two spatial dimensions. We illustrate the principle in the case of domainwalls (DWs) and vortices in two spatial dimensions (relevant results, but for a rotating trap were reported in [32]). As concerns the DW solutions, instead of using the more "standard" circular DWs (between a less repulsive component in the middle of the trap and a more repulsive one forming an outer shell) [6,7], we will use the recently proposed dipolar (i.e., rectilinear) and quadrupolar (i.e., cross-like) DWs of [33]. Once such a stationary state is reached for Eq. (6) (via imaginary time integration), using it as initial condition in Eqs. (1)-(2) produces a time periodic solution of the latter. Such examples are shown in Fig. 3 for the different types of DWs in the case of a magnetic trap potential $V(r)=(1 / 2) \Omega^{2} r^{2}\left(r^{2} \equiv x^{2}+y^{2}\right)$. We should note that the DW can, in principle, exist if the immiscibility condition $\Delta \equiv a_{11} a_{22}-a_{12}^{2} \leq 0$ is satisfied. In particular, the quadrupolar DWs can persist even for $\Delta=0$ (or $g=h=1$ ) and hence the resulting "rotating propeller" solutions are exact time periodic solutions of Eq. (3). In the bottom panel of Fig. 3, a DW cross is shown for the practical case of $a_{11}=1.03, a_{12}=1$ and $a_{22}=0.97$ (these values pertain to two different spin states of $\left.{ }^{87} \mathrm{Rb}[16]\right)$. On the other hand, the dipolar DWs only persist if $\Delta \leq-0.061$, which cannot be satisfied for the ${ }^{87} \mathrm{Rb}$ parameters. In the top panel of Fig. 3 such a dipolar DW is shown, in the limiting case $\Delta=-0.061$ (for $g=1$ and $h=1.03$ ); apparently, the time-periodic, dipolar DWs are only approximate solutions. Similarly to the $1 \mathrm{D}$ results, if $h \neq g$ (or, generally, $\Delta \neq 0$ ), we have found that there is a maximum (minimum) critical $h_{c}\left(\Delta_{c}\right)$ up to which the Rabi oscillations persist [for the bulk of each component]: $h_{c}=1.67\left(\Delta_{c}=-1.78\right)$ for dipolar DWs and $h_{c}=1.28\left(\Delta_{c}=-0.64\right)$ for the quadrupolar DWs.

Finally, one can use a similar construction for a pair of vortex structures with two components (see, e.g., Fig. 4). In this case, we initialize the imaginary time integration, in the absence of the linear coupling, with one component having a vortex centered at $(5,0)$, while the other has a vortex at $(-5,0)$. After the configuration relaxes to the stationary vortex pair solution of Eqs. (1)-(2), we again turn on the linear coupling and obtain a spiral rotation between the vortices resembling a spiral wave. In this case also, there is a critical $h_{c}=1.32\left(\Delta_{c}=-0.74\right)$ beyond which the regularity of Rabi oscillations is destroyed. In this case, the breakup leads to the formation of spiral patterns in the condensate.

Conclusions and Generalizations. In this Letter, we have illustrated the possibility of coupled Bose-Einstein condensates to sustain exact periodic and quasi-periodic patterns in the presence of an experimentally realizable, linear coupling between the components. Similar results should be immediately applicable to linearly coupled optical systems close to the Manakov limit. A unitary transformation, commuting with the nonlinear kernel was identified as the source of such solutions and as a way of "factoring out" the linear coupling by means of timedependent oscillatory behavior with a frequency equal to the strength of the linear coupling. We demonstrated the relevance of this transformation in constructing various solutions in the presence of external potentials such as the optical lattice and the magnetic trap potential. We also illustrated the robustness of the mechanism in demonstrating that the phenomenon persists even for a wide range of parameter values (rather than only for the special yet experimentally relevant case of equal interand intra-species interaction for which it is exact).

Finally, we would like to indicate that this mechanism is not restricted to the particular case of two linearly coupled components, but in fact generalizes to higher numbers of components (e.g., 3 linearly coupled hyperfine states can also be realized in the context of BECs [26]). In this case, the term $\alpha P \psi$ in Eq. (3) should be substituted with $\hat{\alpha} \psi$, where $\hat{\alpha}$ is a symmetric $n \times n$ matrix (with zeros along the diagonal). The unitary transformation then becomes $U(t)=e^{-i t \hat{\alpha}}$; e.g., in the special case where $n=3$ and all off-diagonal elements are identical, then $U(t)=\left[\beta_{i j}\right]$, with diagonal elements $\beta_{j j}=(1 / 3)\left(2 e^{i \alpha t}+e^{-2 i \alpha t}\right)$ and off-diagonal ones $\beta_{i j}=\beta_{j j}+e^{i \alpha t}$.

Acknowledgements This work was supported in part by NSF-DMS-0139093 (BD), NSF-DMS-0204585, 
NSF-CAREER, and the Eppley Foundation for Research (PGK).

[1] F. Dalfovo et al., Rev. Mod. Phys. 71, 463 (1999).

[2] S. Burger et al., Phys. Rev. Lett. 83, 5198(1999); J. Denschlag et al., Science 287, 97 (2000); B.P. Anderson et al., Phys. Rev. Lett. 86, 2926 (2001).

[3] K.E. Strecker et al., Nature 417, 150 (2002); L. Khaykovich et al., Science 296, 1290 (2002).

[4] B.A. Malomed Prog. Opt. 43, 71 (2002); A.V. Buryak et al., Phys. Rep. 370, 63 (2002).

[5] Yu.S. Kivshar and B. Luther-Davies, Phys. Rep. 298, 81 (1998).

[6] T.-L. Ho and V.B. Shenoy, Phys. Rev. Lett. 77, 3276 (1996); H. Pu and N.P. Bigelow, Phys. Rev. Lett. 80, 1130 (1998).

[7] B.D. Esry et al., Phys. Rev. Lett. 78, 3594 (1997).

[8] Th. Busch et al., Phys. Rev. A 56, 2978 (1997); R. Graham and D. Walls, Phys. Rev. A 57, 484 (1998); H. Pu and N.P. Bigelow, Phys. Rev. Lett. 80, 1134 (1998); B.D. Esry and C.H. Greene, Phys. Rev. A 57, 1265 (1998).

[9] M. Trippenbach et al., J. Phys. B 33, 4017 (2000).

[10] S. Coen and M. Haelterman, Phys. Rev. Lett. 87, 140401 (2001).

[11] P. Öhberg and L. Santos, Phys. Rev. Lett. 86, 2918 (2001).

[12] Th. Busch and J.R. Anglin, Phys. Rev. Lett. 87, 010401 (2001).

[13] P.G. Kevrekidis et al., Eur. Phys. J. D 28, 181 (2004).

[14] B. Deconinck et al., J. Phys. A 36, 5431 (2003).

[15] C.J. Myatt et al., Phys. Rev. Lett. 78, 586 (1997).

[16] D.S. Hall et al., Phys. Rev. Lett. 81, 1539 (1998); M.R. Matthews et al., Phys. Rev. Lett. 81, 243 (1998).

[17] D.M. Stamper-Kurn et al., Phys. Rev. Lett. 80, 2027 (1998).

[18] G. Modugno et al., Science 294, 1320 (2001).

[19] M. Mudrich et al., Phys. Rev. Lett. 88, 253001 (2002).

[20] D.S. Hall, personal communication.

[21] P.G. Kevrekidis and D.J. Frantzeskakis, Mod. Phys. Lett. B 18, 173 (2004).

[22] B.A Malomed, Phys. Rev. A 43, 410 (1991).

[23] M.J. Potasek, J. Opt. Soc. Am B 10, 941 (1993).

[24] S.M. Jensen, IEEE J. Quantum Electron. 18, 1580 (1982).

[25] B.A. Malomed et al., Phys. Rev. E 53, 4084 (1996).

[26] M.A. Porter et al., nlin.CD/0401023.

[27] M.R. Matthews et al., Phys. Rev. Lett. 83, 3358 (1999).

[28] S.V. Manakov, Sov. Phys. JETP 38, 248 (1974).

[29] R.M. Bradley et al., nlin.PS/0403043.

[30] F.S. Cataliotti et al., Science 293, 843 (2001); A. Trombettoni and A. Smerzi, Phys. Rev. Lett. 86, 2353 (2001); M. Greiner et al., Appl. Phys. B 73, 769 (2001).

[31] J.C. Bronski et al., Phys. Rev. Lett. 86, 1402 (2001).

[32] J.J. García-Ripoll et al., Phys. Rev. A 66, 021602(R) (2002).

[33] B.A. Malomed et al., "Static and rotating domain-wall crosses in Bose-Einstein condensates", (submitted Phys. Rev. A).
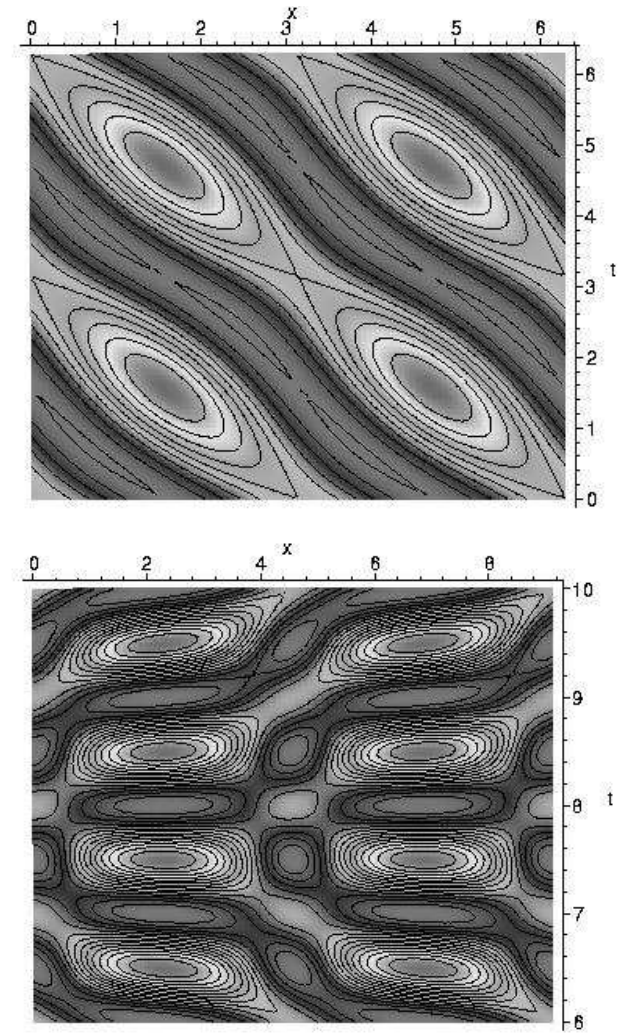

FIG. 1. Top panel: two spatial periods of the time-periodic oscillations of the density $n_{2}$ for the trigonometric solution in the OL potential. Here $g=1, \alpha=1, m=1$, $\omega_{1}=\omega_{2}=\frac{1}{2} m^{2}+B_{1}+B_{2}$, and $V_{0}=1, A_{1}=2$, $A_{2}=V_{0}-A_{1}=1, B_{1}=1, B_{2}=2$. Bottom panel: two spatial periods of a quasiperiodic-in-time oscillation of the density $n_{2}$ for the elliptic function solution given in the text. Here $m=1$, $g=1, \alpha=\pi, \omega_{1}=\frac{1}{2} m^{2}\left(1+k^{2}\right)-A_{2}, \omega_{2}=\frac{1}{2} m^{2}-A_{2}$. Also $V_{0}=1, A_{1}=2, A_{2}=V_{0}+m^{2} k^{2}-A_{1}=1$. 

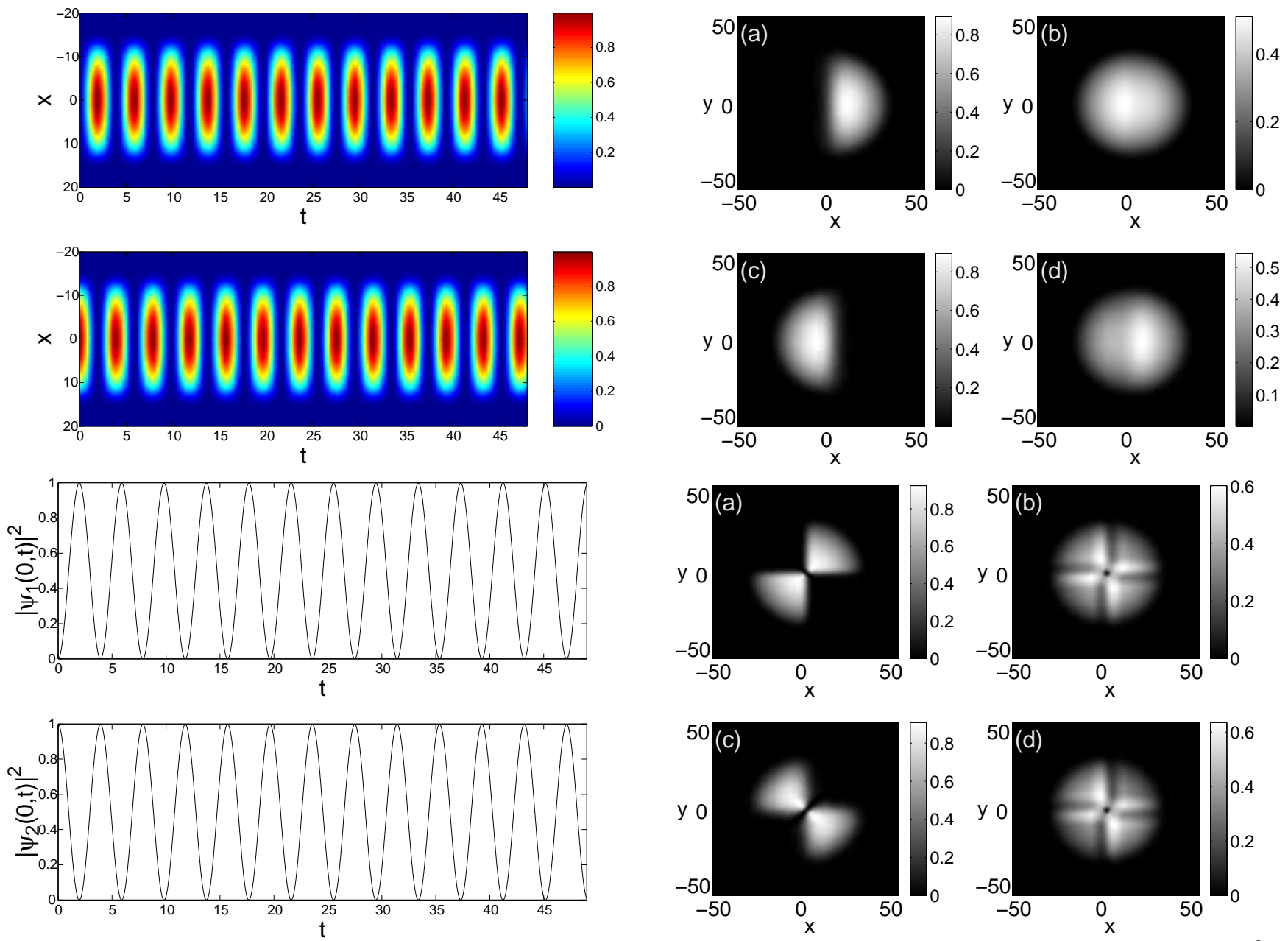

FIG. 3. Top panels: Contour plots of the density $\left|\psi_{1}\right|^{2}$ for
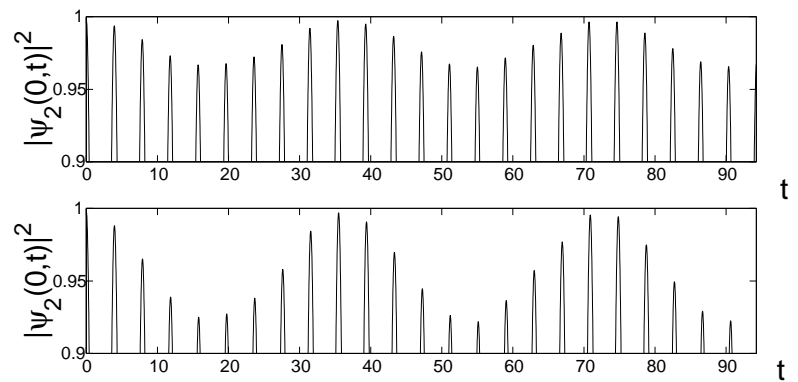

a dipolar DW (the density $\left|\psi_{2}\right|^{2}$ of the other species is complementary to $\left|\psi_{1}\right|^{2}$ ) for $t=0$ (a), $T / 4$ (b), $T / 2(\mathrm{c})$, and $3 T / 4$ (d), with $T=\pi / \alpha \approx 15.7(\alpha=0.2) ; \Omega=0.045, \Delta=-0.061$. The pattern persists for long times with the species "interchanging places". Bottom panels: Same as the top but for the quadrupolar DW with $\Delta=-9 \times 10^{-4}$ (the respective nonlinearity coefficients pertain to ${ }^{87} \mathrm{Rb}$ ). The Rabi oscillation of this sloshing DW gives the impression of a rotating propeller.

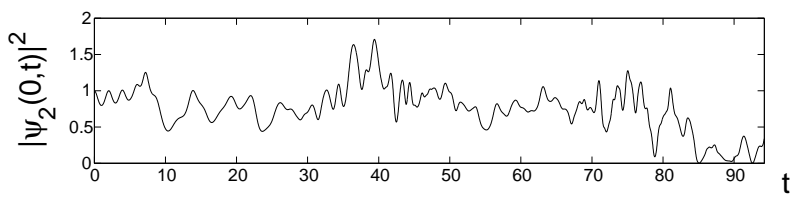

FIG. 2. (Color Online) Top two panels: the two subplots show exact Rabi oscillations of a ground state BEC in the presence of a magnetic trap. The oscillation frequency is given by $\alpha=0.8$. The magnetic potential has a frequency $\Omega=0.1$. The two subplots show the contour of the space-time evolution of the density of each component. Middle two panels: evolution of the density at the center of the magnetic trap i.e., at $x=0$ for the two components. Bottom three panels: evolution at the center of the magnetic trap, for $h \neq g$, namely for $h=1.2$ (top subplot), $h=1.5$ (middle subplot) and $h=2$ (bottom subplot). 

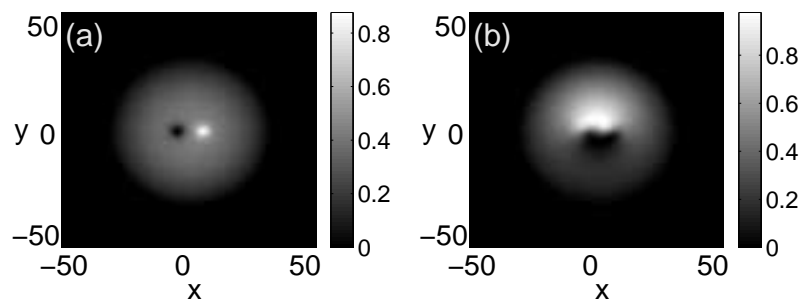

FIG. 4. Top panels: Contour plots of $\left|\psi_{1}\right|^{2}$ for two coupled vortices, initially placed at $x= \pm 5$, for $t=0$ (a), $T / 4$ (b), $T / 2$ (c), and $3 T / 4(\mathrm{~d})$, with $T=\pi / \alpha \approx 15.7(\alpha=0.2)$; $\Omega=0.045, \Delta=-9 \times 10^{-4}\left({ }^{87} \mathrm{Rb}\right)$. The vortices "interchange locations" (in a structure resembling a spiral wave). Bottom panels: Same as the top but for $\Delta=-3(g=1, h=2)$. The configuration breaks up forming spiral patterns.
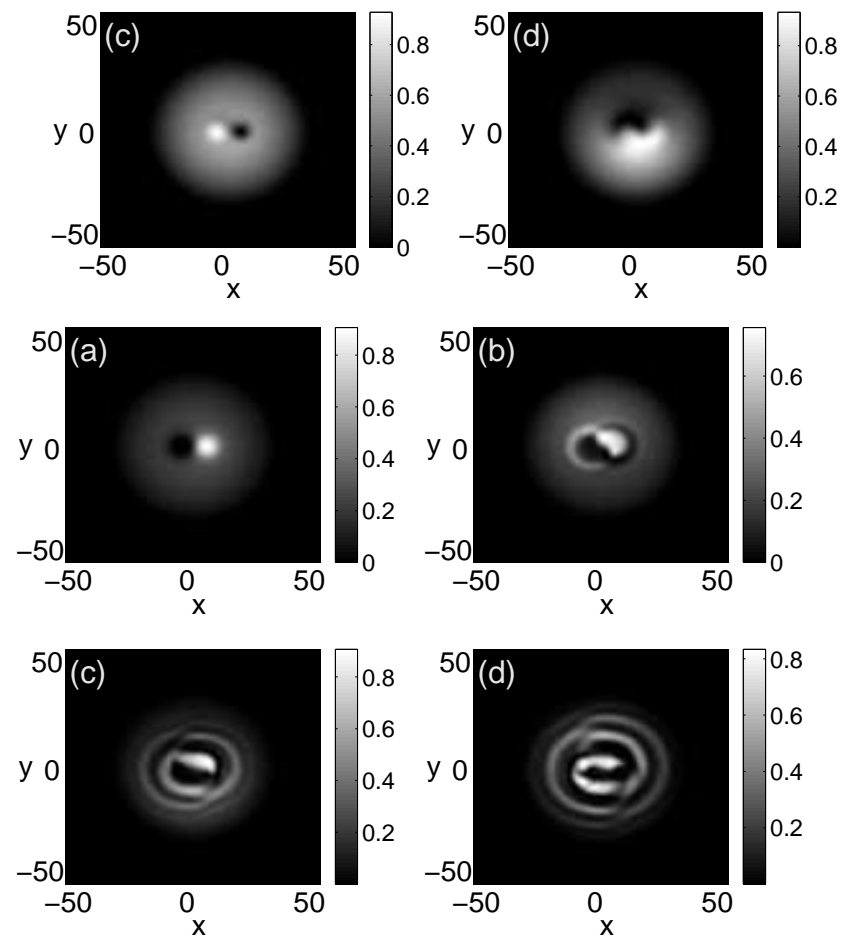